\title{
Aortic Dissection with Haemothorax Mimicking a Stroke
}

\author{
Joana Ricardo Pires ${ }^{1}$, Mariana Teixeira ${ }^{1}$, Margarida Ferreira ${ }^{2}$, Clarinda Neves ${ }^{1}$ \\ ${ }^{1}$ Internal Medicine Department, Centro Hospitalar do Baixo Vouga, Aveiro, Portugal \\ ${ }^{2}$ Radiology Department, Centro Hospitalar do Baixo Vouga, Aveiro, Portugal
}

Received: 13/09/2019

Accepted: 03/10/2019

Published: 04/11/2019

How to cite this article: Pires JR, Teixeira M, Ferreira M, Neves C. Aortic dissection with haemothorax mimicking a stroke. EJCRIM 2019;6: doi:10.12890/2019_001277.

Conflicts of Interests: The Authors declare that there are no competing interest

This article is licensed under a Commons Attribution Non-Commercial 4.0 License

\section{ABSTRACT}

Aortic dissection is a life-threatening clinical emergency and a challenging diagnosis. Depending on its initial location, it may present with several symptoms with the most common being chest pain. We describe the case of a 62-year-old man admitted to the Emergency Department with acute neurological deficits and triaged for the stroke protocol. After unexpected findings on physical examination, other diagnostic hypotheses were evaluated, culminating in the diagnosis of aortic dissection with haemothorax mimicking a stroke.

\section{LEARNING POINTS}

- Aortic dissection is a clinical emergency with a high rate of misdiagnosis; it may present with unusual symptoms depending on its initial location.

- Stroke mimics are diverse non-vascular conditions that can present with acute neurological deficits simulating an acute stroke, some of which are contraindications for thrombolytic treatment.

- An accurate initial physical examination in the emergency department is crucial so less common conditions can be evaluated and fatal thrombolytic treatment avoided.

\section{KEYWORDS}

Aortic dissection, stroke, haemothorax, emergency medicine

\section{CASE DESCRIPTION}

A 62-year-old man with a medical history of cerebrovascular disease, type 2 diabetes, smoking and alcohol abuse, presented to the Emergency Department after the sudden onset of an altered level of consciousness with no chest pain. He was triaged for the stroke protocol. He had been hospitalised 8 months previously due to cryptogenic ischaemic stroke and had been discharged with no neurological deficits.

First vital signs revealed hypotension $(77 / 68 \mathrm{mmHg}$ ), irregular tachycardia with a heart rate of $120 \mathrm{bpm}$, normal body temperature, normal respiratory rate and hyperglycaemia (222 mg/dl). On examination, the patient had a score of 10 on the Glasgow Coma Scale, global aphasia, right gaze palsy with reactive and symmetric pupils, and no apparent motor impairment. Cardiac auscultation revealed irregular cardiac sounds but no murmurs, while pulmonary auscultation revealed absent breath sounds over the left lung field, with dullness on percussion. An electrocardiogram confirmed atrial fibrillation and an arterial blood gas test revealed mild hypoxaemia but with important hyperlactataemia (lactate $3.7 \mathrm{mmol} / \mathrm{l})$.

The stoke protocol was followed. A cerebral CT scan was performed and revealed multifocal vascular lesions and leukoaraiosis but no evidence of recent ischaemia or haemorrhage. Before thrombolytic treatment with alteplase was initiated, a chest x-ray was conducted and showed right deviation of the mediastinum with a prominent aortic arch and left pleural effusion (Fig. 1A). Therefore, it was decided to perform a thoracic CT scan which revealed a dissecting aneurysm of the thoracic aorta, with distal onset at the emergence of the supra-aortic 
vessels (Fig. 1B) and exuberant mural thickening at the level of the aortic arch, with several spontaneously dense laminar areas suggesting haematoma of the aneurysm wall, as well as massive left pleural effusion compatible with haemothorax (Fig. 1C).

Haemodynamic stability was achieved and the patient was transferred to another hospital so vascular surgery could be performed.

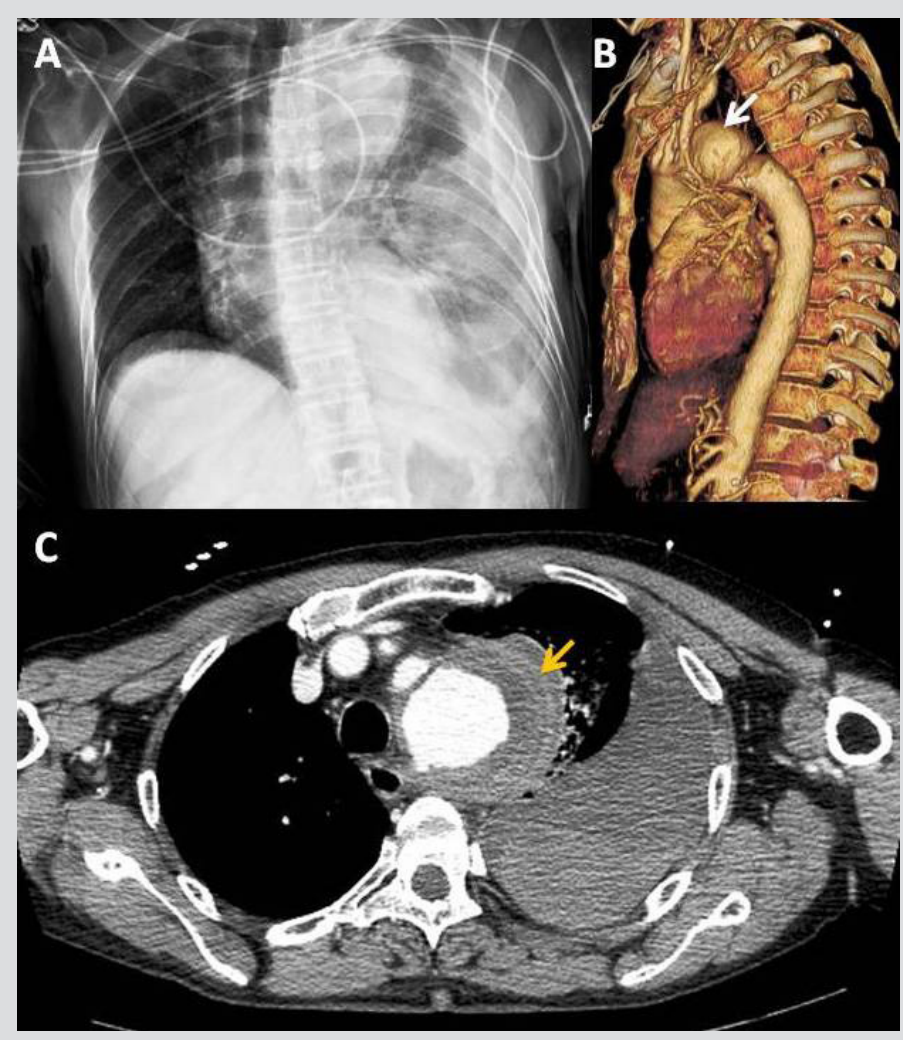

Figure 1. (A) Chest $x$-ray with right deviation of the mediastinum, a prominent aortic arch and left pleural effusion. (B) Aneurysm of the thoracic aorta (white arrow) with distal onset at the emergence of the supra-aortic vessels. (C) Mural thickening at the level of the aortic arch, with several spontaneously dense laminar areas suggesting a haematoma (yellow arrow) of the aneurysm wall, and left pleural effusion compatible with haemothorax

\section{DISCUSSION}

Aortic dissection is a life-threatening clinical emergency and a challenging diagnosis, with an estimated incidence of misdiagnosis of approximately $31 \%^{[1]}$. Alter et al. reported that an emergency physician seeing 4,000 patients a year would diagnose one aortic dissection approximately every 3-4 years ${ }^{[2]}$. Neurological and cardiovascular manifestations in some cases might be the only symptoms instead of chest pain ${ }^{[3]}$. Moreover, if patients present with cortical symptoms such as aphasia or impaired consciousness, they will not be capable of explaining their symptoms, making the diagnosis more difficult.

Ischaemic stroke is also a clinical emergency with a very short window of opportunity for intervention, so an accurate diagnosis with early treatment is paramount for preventing a larger area of irreversible ischaemic brain damage. However, stroke mimics are common and other diverse non-vascular conditions can present with acute neurological deficits simulating an acute stroke.

Some clinical reports described cases of aortic dissection which presented solely with neurological manifestations where diagnosis was only achieved after thrombolytic treatment with fatal consequences such as dissection extension, haemothorax or haemopericardium complications, emphasising the importance of a chest x-ray in the emergency department ${ }^{[4]}$. Unfortunately, clinical assessment of aortic dissection has been removed from recent guidelines. The AHA/ASA 2018 guidelines consider that the value of chest radiographs is unclear in the hyperacute stroke setting in the absence of evidence of acute pulmonary or cardiac disease, and they should not delay fibrinolytic treatment ${ }^{[5]}$. Conversely, Australian guidelines recommend routine imaging of the entire vasculature from the aortic arch to the cerebral vertex to improve diagnosis, recognition of stroke aetiology and prognosis assessment ${ }^{[6]}$. They also state that a chest $x$-ray may be required and may need to be performed as an emergency procedure in certain patients ${ }^{[6]}$. In addition, a Japanese working group developed an algorithm to integrate guidelines for the exclusion of aortic dissection in cases of ischaemic stroke but is still waiting for clinical evaluation to verify its effectiveness ${ }^{[7]}$. 
In our case, multiple findings on initial examination, such as hypotension and no breath sounds in the left lung field, with dullness on percussion, were crucial in raising the suspicion of a stroke mimicked by arterial perfusion deficits. Additionally, the chest x-ray with mediastinal widening and left pleural effusion suggested a haemothorax of vascular origin confirmed later by thoracic CT. Rarely, haemothorax and neurological symptoms are present simultaneously in aortic dissection. In our case, the combined presentation of symptoms was due to the location of the dissection. Haemothorax of vascular origin is usually due to a rupture of the descending thoracic aorta ${ }^{[8]}$, and the neurological symptoms due to rupture of the aortic arch or at the emergence of the supra-aortic vessels. The described aortic dissection mimicked a stroke and caused a significant haemothorax.

In cases with large anterior vessel occlusion symptoms and unexpected findings on physical examination, aortic dissection should be considered. Each centre should employ their fastest available investigation methods, such as CT angiography encompassing the aortic arch, chest x-ray or even ultrasound of the cervical arteries and an echocardiogram. In cases where the time window is short and CT angiography cannot be performed before fibrinolysis, a chest x-ray in the emergency department should be considered.

It is important to promote updated stroke protocols in order to alert clinicians of the importance of excluding stroke mimics with potentially fatal consequences following fibrinolysis such as aortic dissection.

\section{REFERENCES}

1. Asouhidou I, Asteri T. Acute aortic dissection: be aware of misdiagnosis. BMC Res Notes 2009;2:25.

2. Alter SM, Eskin B, Allegra JR. Diagnosis of aortic dissection in emergency department patients is rare. West J Emerg Med 2015;16(5):629-631.

3. Deniz T, Dag E, Tulmac M, Azapoglu B, Alp C. What lies behind the ischemic stroke: aortic dissection? Case Rep Emerg Med 2014;2014:468295.

4. Pastuszak Ż, Stępień A, Kordowska J, Rolewska A, Galbarczyk D. Brain strokes related to aortic aneurysma - the analysis of three cases. Open Med (Wars) 2017;12:58-61.

5. Powers WJ, Rabinstein AA, Ackerson T, Adeoye OM, Bambakidis NC, Becker K, et al. 2018 Guidelines for the Early Management of Patients with Acute Ischemic Stroke: A Guideline for Healthcare Professionals from the American Heart Association/American Stroke Association. Stroke 2018;49:e46-110.

6. The Stroke Foundation. Clinical Guidelines for Stroke Management 2017. Melbourne; 2017.

7. Koga M, Iguchi Y, Ohara T, Tahara Y, Fukuda T, Noguchi T, et al. Acute ischemic stroke as a complication of Stanford type A acute aortic dissection: a review and proposed clinical recommendations for urgent diagnosis. Gen Thorac Cardiovasc Surg 2018;66:439-445.

8. Chermiti F, Abdallah B, Gharsalli H, Kwas H, Taktak S, Chtourou A, et al. Hemothorax revealing aortic aneurism and aortic dissection. Open J Respir Dis 2013;3:113-115. 